\title{
Tabagismo no Brasil: desigualdades regionais e prevalência segundo características ocupacionais
}

\author{
Tobacco smoking in Brazil: regional inequalities \\ and prevalence according to occupational characteristics
}

\begin{abstract}
Aluísio J. D. Barros ${ }^{1}$
Andreia Morales Cascaes ${ }^{1}$

Fernando César Wehrmeister ${ }^{1}$

Jeovany Martínez-Mesa ${ }^{1}$

Ana Maria Baptista Menezes ${ }^{1}$
\end{abstract}

${ }^{1}$ Programa de Pós-

Graduação em

Epidemiologia da

Universidade Federal de

Pelotas. Av. Marechal

Deodoro $1160 / 3^{\circ}$ piso,

Centro. 96020-220 Pelotas

RS. abarros.epi@gmail.com
Abstract This study describes the prevalence of daily tobacco smoking according to sex, age, per capita household income and occupation of residents aged 15 years or more in Brazil and regions using data from the 2008 National Household Sample Survey (PNAD/IBGE). The analysis was adjusted for the sampling design and included 252.768 individuals. Daily smoking prevalence in Brazil was $15.1 \%$, varying from $12.8 \%$ in the North region to $17.4 \%$ in the South region, and it was $62 \%$ higher in men compared to women. Smoking prevalence was inversely proportional to household income, $18.6 \%$ among the poorest $20 \%$ and $11.5 \%$ among the wealthiest $20 \%$. The same trends for gender, age and income were observed in the different regions of Brazil. Daily smoking was 3\% higher among workers compared to non-workers. White collar workers presented a smoking prevalence below 10\%, while blue collar workers had rates above 20\%. The association between smoking and occupation persisted after the adjustment for sex, age and household income. The inequalities found should be considered when developing effective strategies for smoking reduction. The more exposed occupational groups should have priority in the interventions. Key words Tobacco smoking, Prevalence, Health inequalities, Occupation
Resumo O estudo descreveu a prevalência do tabagismo diário segundo sexo, idade, renda domiciliar e ocupação dos moradores de 15 anos ou mais, no Brasil e regiões, baseado nos dados da Pesquisa Nacional por Amostra Domiciliar 2008 (PNAD/IBGE). A análise considerou o desenho da amostra e incluiu 252.768 indivíduos. A prevalência de fumo diário no Brasil foi 15,1\%,variando de $12,8 \%$ na região Norte a $17,4 \%$ na região Sul, sendo 62\% maior nos homens que mulheres. A prevalência de fumo foi inversamente proporcional à renda domiciliar, sendo $18,6 \%$ entre os $20 \%$ mais pobres e $11,5 \%$ entre os $20 \%$ mais ricos. As mesmas tendências para sexo, idade e renda foram observadas nas diferentes regiões do país. O consumo diário de cigarros foi $3 \%$ maior entre os trabalhadores comparados com não trabalhadores. Trabalhadores não manuais apresentaram prevalências de fumo abaixo de $10 \%$, enquanto trabalhadores manuais relataram frequências acima de 20\%. A associação entre tabagismo e ocupação permaneceu após ajuste para sexo, idade e renda. As desigualdades encontradas devem ser consideradas no planejamento e direcionamento de ações efetivas para redução do tabagismo. Os grupos ocupacionais mais expostos deveriam ter prioridade nas intervenções.

Palavras-chave Tabagismo, Prevalência, Desigualdades em saúde, Ocupação 


\section{Introdução}

A Organização Mundial da Saúde (OMS) estima que mais de cinco milhões de mortes ao ano no mundo são decorrentes do tabagismo e espera-se que esse número seja de aproximadamente oito milhões no ano 2030, tornando o tabagismo a principal causa de morte prematura, sendo que $80 \%$ delas ocorrerão em países em desenvolvimento ${ }^{1}$.

Lopez et al. ${ }^{2}$ propuseram um modelo baseado em quatro estágios para descrever a epidemia de tabagismo, definida basicamente a partir da análise da prevalência e mortalidade atribuível. Países da América Latina situam-se em estágios variados desta epidemia. Cuba e Chile apresentam prevalências acima de $40 \%$ para homens e em torno de 30\% para mulheres. Na Argentina, Bolívia, Paraguai, Uruguai, Venezuela, 30\% a 40\% dos homens fumam, enquanto a prevalência em mulheres está entre $20 \%$ e $30 \%{ }^{3}$. No Brasil, a prevalência de fumantes correntes é $17,2 \%$, sendo $21,6 \%$ entre homens e $13,1 \%$ em mulheres e variando de $16,7 \%$ na região sudeste a $19,0 \%$ na região sul ${ }^{4}$. O Brasil encontra-se em estágio intermediário, apresentando maior declínio da prevalência em homens do que mulheres, padrão observado nos países em desenvolvimento, e, prevalência entre mulheres jovens em crescimento maior do que homens da mesma idade, característica de países desenvolvidos ${ }^{5}$.

A redução global do consumo do tabaco no Brasil deve-se em parte ao Programa Nacional de Controle do Tabagismo, considerado como um dos mais abrangentes entre os países em desenvolvimento e cujo foco está voltado para: a proibição da propaganda, a obrigatoriedade das imagens de advertência nas carteiras de cigarro e restrições ao fumo em ambientes fechados de uso coletivo. Apesar dos avanços, vários desafios ainda permanecem, como limitar o acesso ao consumo, aumentar os preços dos produtos do tabaco, melhorar a fiscalização das medidas e reduzir as desigualdade no alcance da efetividade das ações nos diferentes níveis socioeconômicos.

Inquéritos de base populacional realizados em países desenvolvidos têm monitorado as tendências do uso do tabaco segundo ocupação ${ }^{6-9}$. As pesquisas revelam que a prevalência do tabagismo vem se reduzido em todas as categorias de ocupação. No entanto, permanece maior entre trabalhadores manuais comparados com não manuais. Considerando que o local de trabalho representa um espaço ideal para medidas de restrição ao tabagismo, estudos em nível nacional que descrevem o tabagismo segundo categorias de trabalho são importantes para avançar em políticas públicas para controle do tabagismo no ambiente de trabalho ${ }^{10}$. No Brasil, não encontramos estudos dessa natureza.

O suplemento de saúde da Pesquisa Nacional por Amostra Domiciliar (PNAD), realizada em 2008, forneceu informações sobre o tabagismo representativas das macro-regiões, estados e algumas regiões metropolitanas do Brasil. A vigilância nacional sobre prevalência do tabagismo fornece um quadro da variabilidade quanto a questões demográficas, socioeconômicas e de trabalho para cada região e para o país. Desta forma, contribui para a compreensão dos diferentes perfis de morbi-mortalidade por doenças tabaco-relacionadas e para monitoramento de tendências e efetivação de ações orientadas pelas características de subgrupos mais expostos.

Com o presente estudo nos propusemos descrever a prevalência do tabagismo de acordo com sexo, idade, renda domiciliar e características ocupacionais dos moradores de 15 anos ou mais, no Brasil e suas diferentes regiões, com base nos dados da PNAD 2008.

\section{Métodos}

O estudo foi realizado a partir de dados oriundos da Pesquisa Nacional por Amostra de Domicílios (PNAD) e seu suplemento de saúde, do ano de 2008. A PNAD é uma pesquisa realizada pelo Instituto Brasileiro de Geografia e Estatística (IBGE) e tem por finalidade a produção de informações básicas sobre o desenvolvimento socioeconômico do país. É uma pesquisa com periodicidade anual e, desde 1998, a cada cinco anos, é incluído um suplemento de saúde. O plano de amostragem é feito por conglomerados em três estágios (municípios, setores censitários e domicílio). O desenho amostral garante a representatividade para o país, macrorregiões, estados e algumas regiões metropolitanas. Em 2008, a PNAD incluiu 815 municípios, 150.591 unidades domiciliares e 391.868 indivíduos. Maiores informações sobre os aspectos metodológicos e de amostragem da PNAD podem ser obtidos no documento "Notas Técnicas" disponível no sítio do IBGE ${ }^{11}$.

A população de referência do presente estudo foi baseada em indivíduos com 15 anos ou mais, com informação para hábito de tabagismo no módulo geral de saúde e trabalho na semana de referência do estudo, totalizando 252.768 pessoas. Houve também neste ano um módulo espe- 
cial sobre tabagismo, que não foi utilizado no presente trabalho.

Neste artigo foram analisados os dados referentes ao tabagismo obtidos a partir da seguinte pergunta: Atualmente, fuma algum produto de tabaco?. As respostas diariamente, menos que diariamente e não fuma foram dicotomizadas em "sim" para o grupo que fuma diariamente e "não" para não fumantes e os que responderam menos que diariamente. O número médio de cigarros industrializados fumados diariamente também foi avaliado. Fumantes ocasionais foram definidos como aqueles que relataram fumar menos que diariamente.

A prevalência de fumo diário foi descrita segundo sexo, idade, renda domiciliar per capita e características de trabalho, tais como: se a pessoa trabalha, período da jornada de trabalho, carga horária de trabalho, setor do emprego, ocupação de trabalho, atividade de trabalho e local de trabalho.

A idade foi agrupada em seis categorias, de 15 a 19 , de 20 a 29, de 30 a 39, de 40 a 49 , de 50 a 59 e 60 ou mais anos. Como indicador de nível econômico foi utilizada a renda domiciliar per capita mensal, agrupada em quintis para todo o Brasil (para análises globais) e separadamente para cada região geográfica para as análises por região apresentadas nas figuras.

Em relação às características ocupacionais, foram incluídos na análise indivíduos com informação de trabalho para a semana de referência, sendo excluídos os que estiveram afastados ou que trabalhavam para consumo próprio $(\mathrm{N}=8.180)$. Foram classificados como trabalhadores indivíduos que trabalharam na semana de referência. A carga horária de trabalho semanal, em horas, foi dividida em cinco grupos, de 1-20, 21-30, 31-40, 41-50 e 50+ horas. Jornada diurna de trabalho inclui indivíduos cujo horário de trabalho fica integralmente no período entre 5:00h e 22:00h. O setor de emprego também foi dicotomizado em "público" e "privado". Estas duas últimas variáveis aplicam-se apenas aos empregados em empreendimento de atividade não agrícola e permanentes nos serviços auxiliares da atividade agrícola, no trabalho único ou principal da semana de referência $(\mathrm{N}=84.947)$.

A ocupação seguiu a Classificação Brasileira de Ocupações (CBO), reagrupada em 9 categorias, sendo definida como cargo, função, profissão ou ofício exercido pela pessoa. No grupo de dirigentes estão os membros superiores do poder público, de empresas e gerentes em geral. Os profissionais de ciências e artes incluem pesqui- sadores, professores, artistas e religiosos. Os técnicos de nível médio englobam profissionais de nível médio de áreas científicas, da administração e serviços. O grupo administrativo inclui escriturários e trabalhadores de atendimento ao público. Os trabalhadores dos serviços incluem prestação de serviços de transporte, turismo, hotelaria, serviços de saúde, serviços domésticos e outros, excluindo os trabalhadores do comércio. No grupo de vendedores e prestadores de serviços do comércio estão os vendedores do comércio em lojas e mercados, supervisores, repositores e vendedores ambulantes e a domicílio. Na categoria agrícola encontram-se os produtores e trabalhadores agropecuários. Os trabalhadores da produção de bens e serviços industriais são aqueles que trabalham nos processos industriais contínuos, incluindo máquinas, insumos, bens de consumo, alimentos, etc. No mesmo grupo também se encontram os trabalhadores em serviços de reparação e manutenção da área mecânica, polimantenedores, conservação e manutenção. O grupo das forças armadas inclui todos os membros das forças armadas e policiais militares.

Por fim, a variável local de trabalho foi dividida em quatro grupos: fechado e público que inclui as categorias loja, oficina, fábrica, escola, repartição pública, via ou área pública; aberto que incluiu fazenda, sítio, granja, chácara; domicílio para domicílio em que morava ou domicílio do empregador, patrão, sócio ou freguês; e, outro que agrupou veículo automotor e local designado pelo empregador, cliente ou freguês.

Os dados foram analisados no programa estatístico Stata 11.0 (StataCorp., College St., TX, 2009), utilizando o comando svyset para definir os pesos amostrais e conglomerados e o prefixo svy para todas as análises realizadas tendo em vista o desenho amostral. Realizou-se a análise descritiva de todas as variáveis estudadas através de frequências relativas e absolutas. A prevalência do fumo diário com respectivo intervalo de confiança de $95 \%\left(\mathrm{IC}_{95 \%}\right)$ foi calculada segundo sexo, idade, renda domiciliar per capita e características de trabalho para o Brasil e para cada região do país. Foi utilizada regressão Poisson para calcular as razões de prevalências ${ }^{12}$ e os respectivos intervalos de confiança de 95\% ( IC $\left._{95 \%}\right)$ de fumo diário para o Brasil e macro-regiões segundo características de trabalho, através de análise bruta e ajustada. As prevalências ajustadas foram obtidas em um modelo incluindo a variável de interesse e também categorias de quintis de renda domiciliar per capita e categorias de sexo e 
idade (interação entre sexo e idade), esta última variável agrupada como descrito acima. A avaliação de ajuste do modelo incluiu o cálculo da estatística de qualidade de ajuste e a inspeção dos valores ajustados.

\section{Resultados}

O presente estudo avaliou a amostra de indivíduos de 15 anos ou mais, que possuíam informação sobre tabagismo e ocupação, totalizando 252.768 indivíduos, sendo $131.072(51,8 \%)$ do sexo feminino e 121.696 (48,2\%) do sexo masculino.

As prevalências de tabagismo diário por sexo, idade, renda e regiões são apresentadas na Tabela 1. A prevalência geral do fumo diário no Brasil foi de $15,1 \%$, variando de $17,4 \%$ na região Sul a $12,8 \%$ na região Norte. A prevalência de fumo entre os homens foi $62 \%$ maior que entre as mulheres. No geral, a prevalência de fumo entre homens foi $18,8 \%$ e entre as mulheres, $11,6 \%$. Quando analisado por grupos etários, a proporção de fumantes aumenta com a idade até os 59 anos e cai abruptamente entre os idosos (60 anos ou mais). Tendência semelhante é observada para todas as regiões. O consumo diário de cigarros é tanto menor quanto maior a renda domiciliar, com 18,6\% de tabagismo entre os $20 \%$ mais pobres e $11,5 \%$ entre os 20\% mais ricos. A mesma tendência é observada para todas as regiões do país.

A Figura 1 apresenta as prevalências de fumo diário para o Brasil e regiões, segundo grupos etários e sexo. De maneira geral, homens tiveram maiores prevalências de tabagismo diário do que as mulheres em todos os grupos etários. Entre os homens, a maior prevalência de tabagismo encontrada foi no grupo dos 50 a 59 anos de idade e para as mulheres, o pico foi dos 40 aos 49 anos de idade.

Na Figura 2 apresenta-se uma análise gráfica das prevalências de tabagismo diário no Brasil e regiões, segundo quintis de renda domiciliar per capita e sexo. No Brasil e em todas as regiões, a proporção de fumantes segue tendência de redução com o aumento da renda. O fumo na região Sul é maior do que a média apresentada para o Brasil e demais regiões, em ambos os sexos. Em geral, as diferenças por região se mantêm, apesar da redução com o aumento da renda.

Em relação aos fumantes ocasionais (dados não apresentados em tabelas), a prevalência no Brasil (tendo como denominador toda a amos- tra) foi de $2,1 \%\left(\operatorname{IC}_{95 \%} 2,0 ; 2,2\right)$, sendo $3,8 \%$, $\left(\mathrm{IC}_{95 \%} 3,5 ; 4,1\right), 2,5 \%(2,3 ; 2,6), 1,7 \%\left(\mathrm{IC}_{95 \%} 1,6\right.$; $1,8), 1,9 \%\left(\mathrm{IC}_{95 \%} 1,7 ; 2,0\right)$ e, $1,8 \%\left(\mathrm{IC}_{95 \%} 1,6 ; 2,0\right)$, nas regiões Norte, Nordeste, Sudeste, Sul e Centro-Oeste, respectivamente. Quando analisado segundo sexo, o consumo ocasional de cigarros no Brasil foi mais prevalente entre indivíduos de sexo masculino $\left(2,7 \%, \mathrm{IC}_{95 \%} 2,5 ; 2,8\right)$, contra $1,6 \%$ $\left(\mathrm{IC}_{95 \%} 1,5 ; 1,7\right)$ entre as mulheres. Em relação aos quintis de renda domiciliar per capita, a proporção de fumantes ocasionais foi mais frequente entre os mais pobres, sendo 3,1\% ( $\left.\mathrm{IC}_{95 \%} 2,9 ; 3,3\right)$, 2,4\% ( $\left.\mathrm{IC}_{95 \%} 2,2 ; 2,6\right), 2,1 \%\left(\mathrm{IC}_{95 \%} 1,9 ; 2,2\right), 1,8 \%$ $\left(\mathrm{IC}_{95 \%} 1,7 ; 2,0\right), 1,6 \%\left(\mathrm{IC}_{95 \%} 1,5 ; 1,7\right)$ respectivamente do mais pobre para o mais rico.

Os dados referentes à distribuição do fumo diário no Brasil, assim como as razões de prevalências e a média de cigarros fumados segundo variáveis ocupacionais estão na Tabela 2. Entre as pessoas que trabalham, 16,5\% ( IC $_{95 \%} 16,2$; $16,8)$ fumam diariamente comparadas com $12,9 \%\left(\mathrm{IC}_{95 \%} 12,5 ; 13,2\right)$ das que não trabalham. A prevalência do uso diário de cigarros foi 1,03 $\left(\mathrm{IC}_{95 \%} 1,00 ; 1,06\right)$ vezes maior entre os trabalhadores comparado com os não trabalhadores, após o ajuste por sexo, idade e renda $(\mathrm{p}<0,025)$. Observaram-se diferenças importantes entre categorias ocupacionais. Membros das forças armadas, profissionais das ciências e das artes e os administradores foram os que apresentaram as prevalências de fumo mais baixas (abaixo de 10\%). Em contraste, trabalhadores manuais de produção de bens, manutenção e reparos e os de atividade agrícola tiveram prevalências de fumo acima de $20 \%$. Após ajuste para sexo, idade e renda o efeito de ocupação diminuiu, sendo a maior redução para o grupo de ocupações agrícolas, que teve sua razão de prevalências caindo de 1,75 para 1,52 , em relação ao grupo de dirigentes. De outro lado, os profissionais das ciências e artes apresentaram um risco ajustado de tabagismo $13 \%$ menor que o grupo de dirigentes. É curioso que a média de cigarros fumados não acompanhou a prevalência de fumo no grupo. A maior média foi encontrada entre militares, justamente o grupo de menor prevalência de fumo. E a menor média entre ocupações agrícolas, grupo de maior prevalência. $\mathrm{O}$ diagnóstico dos modelos ajustados não mostrou qualquer problema, sendo todos os testes de qualidade de ajuste não significantes, e todos os valores preditos pelos modelos Poisson pertencentes ao intervalo $[0-1]$. 
Tabela 1. Prevalência de tabagismo diário no Brasil ( $\mathrm{N}=252.768)$ e macro regiões segundo sexo, idade e renda per capita domiciliar em moradores de 15 anos ou mais. Pesquisa Nacional por Amostra Domiciliar - PNAD/IBGE, 2008.

\begin{tabular}{|c|c|c|c|c|c|c|}
\hline \multirow{3}{*}{ Variáveis } & \multicolumn{6}{|c|}{ Prevalência de Tabagismo Diário } \\
\hline & \multicolumn{2}{|r|}{ Brasil } & \multicolumn{2}{|r|}{ Norte } & \multicolumn{2}{|r|}{ Nordeste } \\
\hline & $\mathbf{N}$ & \% (IC95\%) & $\mathbf{N}$ & \% (IC95\%) & $\mathbf{N}$ & \% (IC95\%) \\
\hline Todos & 37.054 & $15,1(14,8 ; 15,4)$ & 3.967 & $12,8(11,9 ; 13,7)$ & 10.638 & $13,9(13,4 ; 14,4)$ \\
\hline \multicolumn{7}{|l|}{ Sexo } \\
\hline Feminino & 14.633 & $11,6(11,3 ; 11,9)$ & 1.314 & $8,4(7,6 ; 9,2)$ & 4.178 & $10,3(9,8 ; 10,8)$ \\
\hline Masculino & 22.241 & $18,8(18,5 ; 19,2)$ & 2.653 & $17,2(16,1 ; 18,4)$ & 6.460 & $17,7(17,1 ; 18,3)$ \\
\hline \multicolumn{7}{|l|}{ Idade } \\
\hline 15 a 19 & 1.547 & $4,9(4,6 ; 5,3)$ & 227 & $4,8(4,0 ; 5,6)$ & 427 & $4,0(3,5 ; 4,4)$ \\
\hline 20 a 29 & 7.183 & $12,3(11,9 ; 12,6)$ & 902 & $10,4(9,4 ; 11,5)$ & 1.903 & $9,5(9,0 ; 10,1)$ \\
\hline 30 a 39 & 7.606 & $16,0(15,5 ; 16,4)$ & 950 & $14,7(13,3 ; 16,1)$ & 2.065 & $14,0(13,2 ; 14,8)$ \\
\hline 40 a 49 & 9.359 & $21,5(21,0 ; 22,1)$ & 889 & $18,6(17,1 ; 20,1)$ & 2.773 & $21,9(20,8 ; 23,0)$ \\
\hline 50 a 59 & 6.807 & $21,8(21,2 ; 22,4)$ & 573 & $17,7(15,8 ; 19,6)$ & 1.875 & $21,4(20,3 ; 22,5)$ \\
\hline 60 ou mais & 4.552 & $13,1(12,6 ; 13,5)$ & 426 & $13,5(11,7 ; 15,2)$ & 1.595 & $16,0(15,0 ; 16,9)$ \\
\hline \multicolumn{7}{|c|}{ Renda em quintis } \\
\hline 1 (menor) & 9.520 & $18,6(18,0 ; 19,1)$ & 1.037 & $17,4(15,4 ; 19,4)$ & 2.557 & $18,3(17,5 ; 19,2)$ \\
\hline 2 & 8.098 & $16,5(16,0 ; 17,0)$ & 855 & $14,4(13,0 ; 15,7)$ & 2.262 & $15,3(14,4 ; 16,3)$ \\
\hline 3 & 7.121 & $15,3(14,9 ; 15,7)$ & 755 & $12,8(11,3 ; 14,2)$ & 2.135 & $14,1(13,4 ; 15,0)$ \\
\hline 4 & 6.101 & $13,8(13,3 ; 14,2)$ & 689 & $11,4(10,4 ; 12,4)$ & 1.928 & $13,1(12,3 ; 13,9)$ \\
\hline 5 (maior) & 5.021 & $11,5(11,1 ; 11,9)$ & 512 & $8,2(7,4 ; 9,0)$ & 1.520 & $8,8(8,2 ; 9,4)$ \\
\hline
\end{tabular}

\begin{tabular}{|c|c|c|c|c|c|c|}
\hline \multirow{3}{*}{ Variáveis } & \multicolumn{6}{|c|}{ Prevalência de Tabagismo Diário } \\
\hline & \multicolumn{2}{|r|}{ Sudeste } & \multicolumn{2}{|r|}{ Sul } & \multicolumn{2}{|c|}{ Centro-Oeste } \\
\hline & $\mathbf{N}$ & $\%(\mathrm{IC} 95 \%)$ & $\mathbf{N}$ & $\%($ IC95\%) & $\mathbf{N}$ & $\%(\mathrm{IC} 95 \%)$ \\
\hline Todos & 11.699 & $15,5(15,2 ; 15,9)$ & 6.769 & $17,4(16,8 ; 18,0)$ & 3.981 & $14,7(13,9 ; 15,4)$ \\
\hline \multicolumn{7}{|l|}{ Sexo } \\
\hline Feminino & 4.812 & $12,3(11,9 ; 12,7)$ & 2.834 & $13,8(13,1 ; 14,5)$ & 1.495 & $10,6(10,0 ; 11,3)$ \\
\hline Masculino & 6.887 & $19,0(18,5 ; 19,5)$ & 3.935 & $21,2(20,4 ; 22,0)$ & 2.486 & $18,9(17,9 ; 19,9)$ \\
\hline \multicolumn{7}{|l|}{ Idade } \\
\hline 15 a 19 & 424 & $5,2(4,6 ; 5,8)$ & 262 & $5,9(5,1 ; 6,7)$ & 207 & $6,1(5,1 ; 7,1)$ \\
\hline 20 a 29 & 2.142 & $13,1(12,5 ; 13,6)$ & 1.382 & $16,6(15,6 ; 17,6)$ & 854 & $12,9(12,0 ; 13,9)$ \\
\hline 30 a 39 & 2.363 & $16,7(16,0 ; 17,4)$ & 1.379 & $19,1(17,9 ; 20,2)$ & 849 & $14,8(13,7 ; 16,0)$ \\
\hline 40 a 49 & 3.106 & $22,1 \quad(21,3 ; 22,9)$ & 1.612 & $21,6(20,4 ; 22,8)$ & 979 & $19,6(18,2 ; 21,0)$ \\
\hline 50 a 59 & 2.346 & $22,2(21,2 ; 23,1)$ & 1.359 & $23,5(22,3 ; 24,8)$ & 654 & $20,0(18,4 ; 21,8)$ \\
\hline 60 ou mais & 1.318 & $11,1(10,5 ; 11,8)$ & 775 & $13,7(12,7 ; 14,7)$ & 438 & $13,7(12,5 ; 14,9)$ \\
\hline \multicolumn{7}{|c|}{ Renda em quintis } \\
\hline 1 (menor) & 2.931 & $19,5(18,7 ; 20,4)$ & 1.738 & $24,0(22,7 ; 25,3)$ & 1.044 & $20,2(19,0 ; 21,5)$ \\
\hline 2 & 2.651 & $16,3(15,5 ; 17,1)$ & 1.406 & $19,1(17,9 ; 20,3)$ & 855 & $16,9(15,6 ; 18,2)$ \\
\hline 3 & 2.016 & $15,6(14,8 ; 16,3)$ & 1.324 & $16,8(15,7 ; 18,0)$ & 777 & $15,0(13,8 ; 16,2)$ \\
\hline 4 & 1.905 & $13,9(13,3 ; 14,6)$ & 1.168 & $14,8(13,8 ; 15,8)$ & 610 & $11,7(10,6 ; 12,9)$ \\
\hline 5 (maior) & 1.642 & $12,3(11,7 ; 13,0)$ & 961 & $12,5(11,6 ; 13,5)$ & 553 & $9,7(8,8 ; 10,6)$ \\
\hline
\end{tabular}

\section{Discussão}

O tabagismo é considerado um problema de saúde pública e está relacionado com 50 diferentes doenças incapacitantes ocasionando em média 200 mil mortes por ano no Brasil, ultrapassando o somatório das mortes por alcoolismo, AIDS, acidentes de trânsito, homicídios e suicídios ${ }^{13}$. Além disso, o tabagismo é responsável por 7,7\% (equivalente a cerca de R\$340 milhões) dos custos de todas as internações e procedimentos de quimioterapia pagos pelo Sistema Único de Saú- 


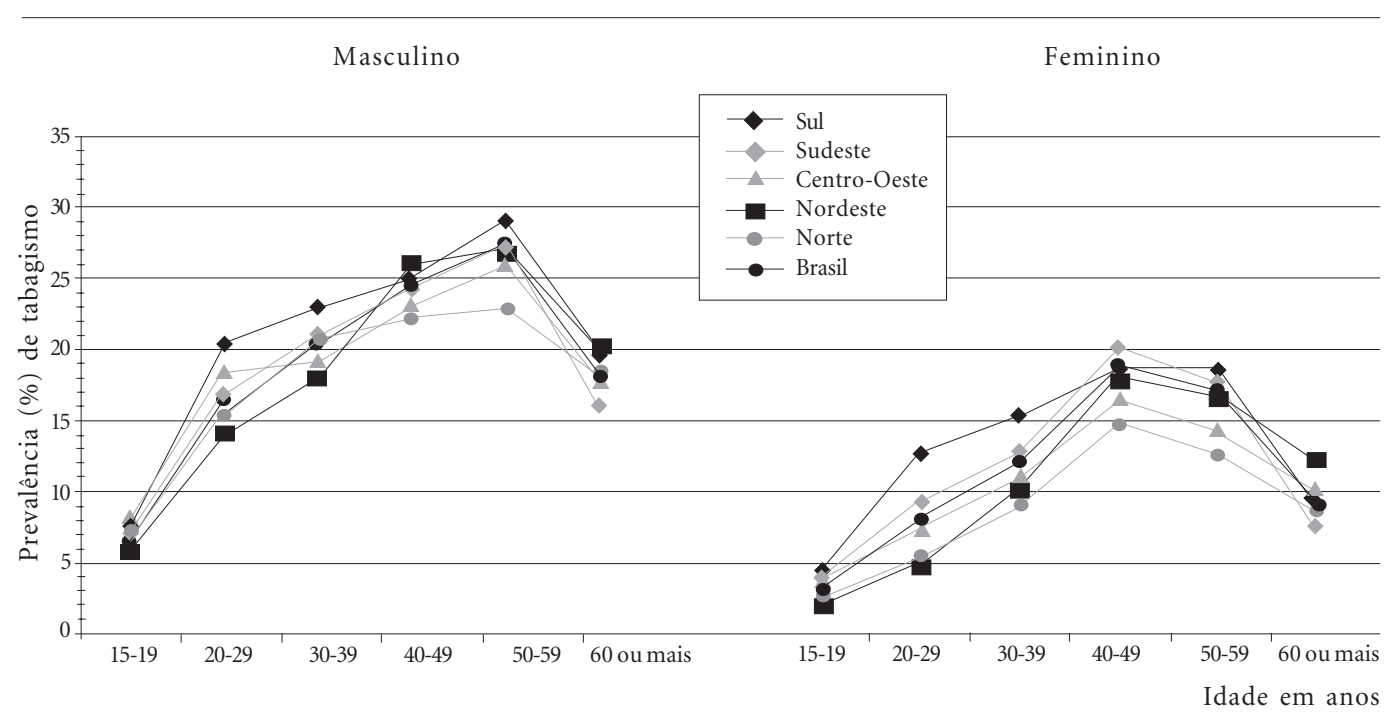

Figura 1. Prevalência de tabagismo diário no Brasil e macro regiões segundo sexo e grupos etários de moradores de 15 anos ou mais. Pesquisa Nacional por Amostra Domiciliar - PNAD, 2008

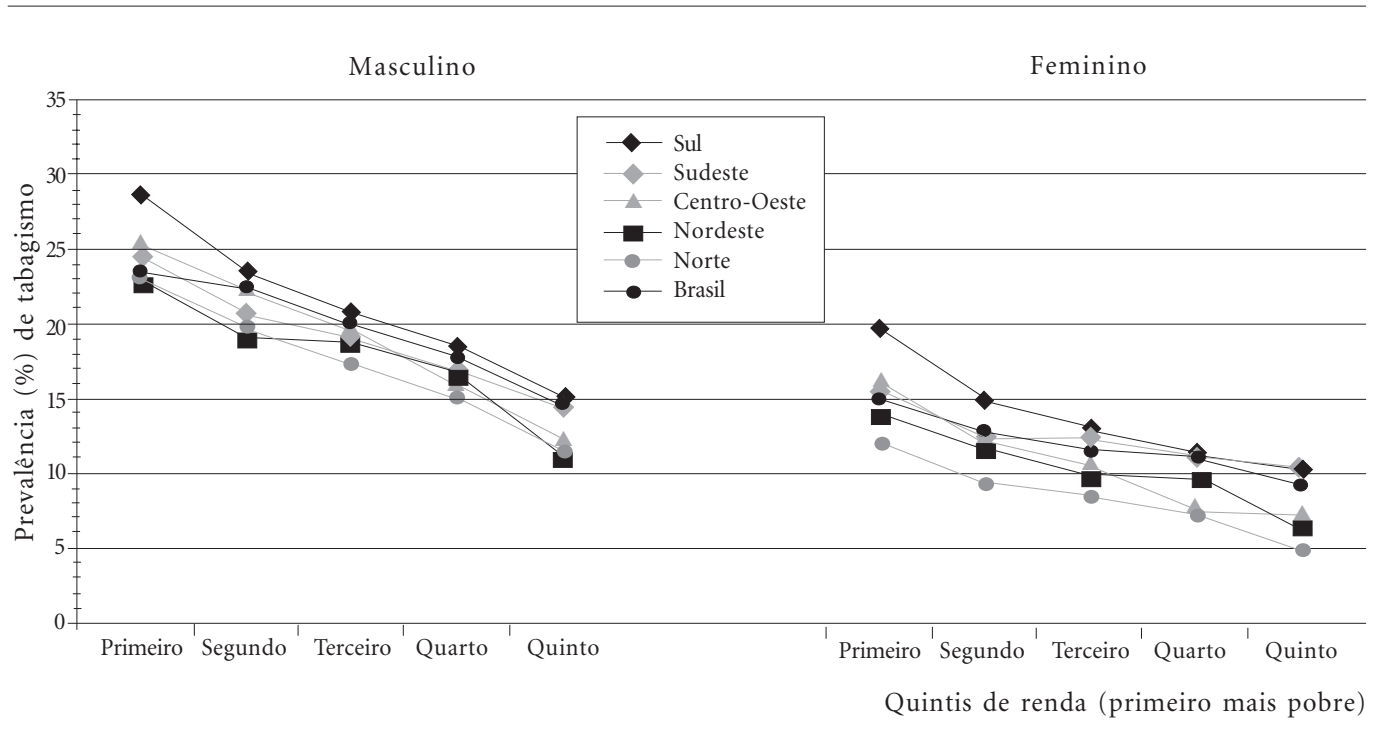

Figura 2. Prevalência de tabagismo diário no Brasil e macro regiões segundo sexo e quintis de renda domiciliar per capita de moradores de 15 anos ou mais. Pesquisa Nacional por Amostra Domiciliar - PNAD, 2008.

de (SUS) para enfermidades relacionadas ao aparelho respiratório, neoplasias e doenças do aparelho circulatório em indivíduos acima de 35 anos de idade ${ }^{14}$.

Pesquisas com a magnitude da PNAD podem contribuir de maneira significativa para a compreensão do panorama de problemas de saúde pública no Brasil, como o tabagismo, ao disponibilizar informações que possam subsidi- ar a formulação de políticas públicas, o que reforça a importância da utilização desses dados e de estudos de nível nacional sobre o tema ${ }^{15}$.

Apesar de ser uma pesquisa de grande abrangência e com metodologia cuidadosa, análises provenientes dos resultados da PNAD não estão isentas de limitações. Por se tratar de um delineamento transversal, não foi possível inferir causalidade às associações encontradas no presente 
Tabela 2. Prevalência de tabagismo diário, razão de prevalências bruta e ajustada e média de cigarros diários fumados segundo características de trabalho* de moradores de 15 anos ou mais no Brasil. Pesquisa Nacional por Amostra Domiciliar - PNAD/IBGE, 2008.

\begin{tabular}{|c|c|c|c|c|c|}
\hline \multirow[t]{2}{*}{ Variáveis } & \multirow{2}{*}{$\begin{array}{l}\mathrm{N} \text { absoluto de } \\
\text { indivíduos }\end{array}$} & \multirow{2}{*}{$\begin{array}{l}\text { Prevalência de } \\
\text { fumo diário } \\
\%\left(\mathrm{IC}_{95 \%}\right)\end{array}$} & \multicolumn{2}{|c|}{ Razão de prevalências } & \multirow{2}{*}{$\begin{array}{c}\text { Cigarros } \\
\text { fumados } \\
\text { diariamente } \\
\text { Média }\left(\mathrm{IC}_{95 \%}\right)\end{array}$} \\
\hline & & & Bruta & Ajustada $^{* *}$ & \\
\hline Trabalha & & $\mathrm{p}<0,001$ & $\mathrm{p}<0,001$ & $\mathrm{p}<0,025$ & $\mathrm{p}<0,001$ \\
\hline Não & 99.275 & $12,9(12,5 ; 13,2)$ & 1,00 & 1,00 & $14,4(14,1 ; 14,7)$ \\
\hline Sim & 153.493 & $16,5(16,2 ; 16,8)$ & $1,28(1,25 ; 1,32)$ & $1,03(1,00 ; 1,06)$ & $15,0(14,8 ; 15,2)$ \\
\hline $\begin{array}{l}\text { Carga horária de trabalho } \\
\text { (horas semanais) }\end{array}$ & & $\mathrm{p}^{* * *}<0,001$ & $\mathrm{p}^{* * *}<0,001$ & $\mathrm{p}^{* * *}<0,001$ & $\mathrm{p}^{* * *}<0,001$ \\
\hline 1 a 20 & 18.386 & $13,0(12,5 ; 13,7)$ & 1,00 & 1,00 & $14,4(13,8 ; 14,9)$ \\
\hline 21 a 30 & 17.088 & $14,7(14,0 ; 15,4)$ & $1,13(1,06 ; 1,20)$ & $1,08(1,02 ; 1,14)$ & $13,9(13,5 ; 14,4)$ \\
\hline 31 a 40 & 48.939 & $16,0(15,5 ; 16,4)$ & $1,22(1,16 ; 1,29)$ & $1,13(1,08 ; 1,19)$ & $14,8(14,5 ; 15,1)$ \\
\hline 41 a 50 & 51.236 & $17,7(17,3 ; 18,1)$ & $1,36(1,29 ; 1,43)$ & $1,21(1,15 ; 1,28)$ & $14,9(14,6 ; 15,2)$ \\
\hline 51 ou mais & 17.844 & $19,8(19,1 ; 20,5)$ & $1,52(1,43 ; 1,60)$ & $1,27(1,20 ; 1,35)$ & $16,9(16,4 ; 17,3)$ \\
\hline $\begin{array}{l}\text { Jornada diurna de } \\
\text { trabalho }^{* * * *}\end{array}$ & & $\mathrm{p}<0,001$ & $\mathrm{p}<0,001$ & $\mathrm{p}=0,021$ & $\mathrm{p}=0,044$ \\
\hline Sim & 76.407 & $14,3(14,0 ; 14,7)$ & 1,00 & 1,00 & $14,7(14,5 ; 15,0)$ \\
\hline Não & 8.540 & $17,5(16,6 ; 18,4)$ & $1,23(1,16 ; 1,30)$ & $1,07(1,01 ; 1,13)$ & $15,5(14,9 ; 16,2)$ \\
\hline Setor do emprego ${ }^{* * * *}$ & & $\mathrm{p}<0,001$ & $\mathrm{p}<0,001$ & $\mathrm{p}<0,001$ & $p=0,272$ \\
\hline Público & 18.540 & $11,8(11,3 ; 12,4)$ & 1,00 & 1,00 & $14,6(14,0 ; 15,1)$ \\
\hline Privado & 66.407 & $15,4(15,0 ; 15,8)$ & $1,31(1,25 ; 1,38)$ & $1,35(1,28 ; 1,42)$ & $14,9(14,6 ; 15,1)$ \\
\hline Ocupação de trabalho & & $\mathrm{p}<0,001$ & $\mathrm{p}<0,001$ & $\mathrm{p}<0,001$ & $\mathrm{p}<0,001$ \\
\hline Forças armadas & 1.339 & $8,4(6,5 ; 10,1)$ & $0,67(0,54 ; 0,85)$ & $0,67(0,54 ; 0,85)$ & $16,3(16,0 ; 16,6)$ \\
\hline Ciências/artes & 10.999 & $9,4(8,7 ; 10,1)$ & $0,75(0,68 ; 0,83)$ & $0,87(0,78 ; 0,96)$ & $14,9(14,3 ; 15,5)$ \\
\hline Administrativo & 14.616 & $9,5(8,9 ; 10,1)$ & $0,76(0,69 ; 0,83)$ & $0,99(0,90 ; 1,08)$ & $13,2(12,6 ; 13,8)$ \\
\hline Técnicos de nível médio & 11.595 & $11,0(10,4 ; 11,7)$ & $0,88(0,81 ; 0,96)$ & $0,98(0,89 ; 1,07)$ & $14,7(14,1 ; 15,4)$ \\
\hline Dirigentes & 8.246 & $12,5(11,6 ; 13,4)$ & 1,00 & 1,00 & $17,6(16,6 ; 18,5)$ \\
\hline $\begin{array}{l}\text { Vendedores e serviços } \\
\text { do comércio }\end{array}$ & 15.473 & $12,8(12,2 ; 13,5)$ & $1,02(0,94 ; 1,11)$ & $1,13(1,04 ; 1,23)$ & $15,1(14,5 ; 15,7)$ \\
\hline $\begin{array}{l}\text { Trabalhadores dos } \\
\text { serviços }\end{array}$ & 32.012 & $18,0(17,5 ; 18,5)$ & $1,44(1,34 ; 1,55)$ & $1,53(1,42 ; 1,65)$ & $14,2(13,9 ; 14,5)$ \\
\hline $\begin{array}{l}\text { Produção de bens/ } \\
\text { serviços e reparação/ } \\
\text { manutenção }\end{array}$ & 37.604 & $21,1(20,6 ; 21,6)$ & $1,68(1,57 ; 1,81)$ & $1,53(1,42 ; 1,65)$ & $15,1(14,5 ; 15,7)$ \\
\hline Agrícola & 21.589 & $21,9(20,1 ; 22,7)$ & $1,75(1,62 ; 1,89)$ & $1,52(1,40 ; 1,65)$ & $12,9(12,3 ; 13,4)$ \\
\hline Local de trabalho & & $\mathrm{p}<0,001$ & $\mathrm{p}<0,001$ & $\mathrm{p}<0,001$ & $\mathrm{p}<0,001$ \\
\hline Fechado/público & 95.794 & $14,1(13,8 ; 14,4)$ & 1,00 & 1,00 & $15,2(14,9 ; 15,4)$ \\
\hline Aberto & 18.002 & $21,9(21,0 ; 22,7)$ & $1,55(1,49 ; 1,62)$ & $1,20(1,15 ; 1,26)$ & $12,9(12,3 ; 13,5)$ \\
\hline Domicilio & 23.320 & $17,5(16,9 ; 18,1)$ & $1,24(1,19 ; 1,29)$ & $1,25(1,20 ; 1,31)$ & $14,4(14,0 ; 14,8)$ \\
\hline Outro & 13.450 & $24,4(23,6 ; 25,3)$ & $1,74(1,67 ; 1,80)$ & $1,37(1,31 ; 1,42)$ & $17,0(16,6 ; 17,5)$ \\
\hline
\end{tabular}

"Características de trabalho na semana de referência do inquérito ( $\mathrm{N}=153.493$ trabalhadores)

${ }^{* *}$ Razão de prevalências ajustada por sexo, idade e renda domiciliar per capita. ${ }^{* * *}$ Valores-p utilizando teste de tendência linear. ${ }^{* * * *}$ Referem-se apenas ao empregado em empreendimento de atividade não agrícola e ao empregado permanente nos serviços auxiliares da atividade agrícola no trabalho único ou principal da semana de referência e que trabalhou na semana de referência $(\mathrm{N}=84.947)$.

estudo. A variabilidade de definições do tabagismo e as distintas metodologias utilizadas em outros estudos possivelmente limitaram a comparação com a nossa pesquisa.

Segundo os inquéritos nacionais da Pesquisa Nacional de Saúde e Nutrição e da Pesquisa de
Saúde Mundial, realizados no Brasil, a prevalência global de tabagismo em adultos acima de 18 anos passou de 34,8\% em 1989 para 22,4\% em $2003^{16}$. Contudo, este declínio ocorreu principalmente entre mais jovens ( $<35$ anos) e mais idosos ( $\geq 65$ anos), sendo maior nos homens (de 
$39,4 \%$ para $27,1 \%)$ do que nas mulheres $(23,9 \%$ para $18,4 \%$ ) e naqueles com melhor condição socioeconômica (de $28,7 \%$ para $15,9 \%$ ) do que com pior condição (de $40,9 \%$ para $29,0 \%)^{16}$. O nosso estudo demonstra que a prevalência do fumo continua em decréscimo, mantendo a tendência de maior redução no sexo masculino (agora com prevalência de $18,8 \%$ ), naqueles com melhor renda $(11,5 \%)$ e nos mesmos grupos etários de avaliações prévias, sugerindo que políticas para prevenção de iniciação e controle do tabagismo foram mais efetivas nesses grupos.

O inquérito nacional da Pesquisa Nacional de Saúde do Escolar (PENSE) ${ }^{17}$, realizado no Brasil em 2009, investigou o tabagismo nos escolares do país. O estudo revelou uma prevalência de fumo diário de 6,3\% em escolares de 13 a 15 anos, pouco acima da encontrada nos adolescentes de 15 a 19 anos da PNAD, em 2008. Adicionalmente, a experimentação do cigarro foi maior entre estudantes de escolas públicas $(25,7 \%)$ em relação aos de escola privada $(18,3 \%)^{17}$, o que possivelmente refletiu a condição socioeconômica desses alunos.

O Brasil é um país de grandes diversidades sociais, econômicas e culturais que podem repercutir nos padrões de consumo do tabaco. A prevalência do fumo diário encontrada em nosso estudo foi maior nas regiões Sul e Sudeste. Os achados concordam com os resultados encontrados das pesquisas do Sistema de Vigilância de Fatores de Risco e Proteção para Doenças Crônicas por Inquérito Telefônico (VIGITEL) que vem monitorando entre outros agravos, o tabagismo, na popu-lação adulta das 26 capitais brasileiras e Distrito Federal. Segundo o VIGITEL, a prevalência global de fumo diário caiu de 16,2\% em 2006 para 15,5\% em 2009. Foram verificadas também diferenças regionais quanto ao consumo diário do tabaco, que variou de $9,7 \%$ em Maceió e 10,0\% em Salvador a 19,4\% em Porto Alegre e 20,9\%, em São Paulo ${ }^{18}$.

O presente estudo também investigou a associação entre tabagismo e trabalho. Resultados provenientes de inquéritos populacionais em países desenvolvidos como National Health Interview Survey (NHIS), os Suplementos de Tabagismo do Current Population Survey (CPS), nos Estados Unidos da América (EUA) e, o National Health Surveys (NHS), na Austrália têm monitorado as prevalências de tabagismo entre as diferentes categorias de ocupação $0^{7-10}$. Nossos resultados vêm ao encontro das tendências observadas nos estudos americanos e australianos, apesar do fumo diário no Brasil ser menos frequen- te. Assim como nos países mencionados, a prevalência de tabagismo em trabalhadores no Brasil foi maior naqueles indivíduos que ocupavam cargos com exigência de menor nível de escolaridade e maior esforço braçal. A frequência do consumo de cigarros no Brasil chega a ser duas vezes maior entre trabalhadores agrícolas $(21,9 \%)$ e da produção de bens e serviços, reparação e manutenção $(21,1 \%)$ comparados com os profissionais qualificados da área de ciências e artes (9,4\%). Nos EUA, por exemplo, a prevalência de fumantes excedeu $35 \%$ nas categorias ocupacionais de construção $(38,8 \%)$, silvicultura e pesca $(38,7 \%)$, alimentação $(38,5 \%)$ e operação de equipamentos $(35,2 \%)$, enquanto em profissionais da área da saúde encontrou-se apenas 5,0\% de fumantes diários ${ }^{9}$. Entre os australianos, a prevalência de fumo diário em trabalhadores foi de $25,0 \%$, sendo mais frequente na categoria de trabalhadores manuais $(39,8 \%)$ e área de transporte $(36,9 \%)$ e menos em profissionais qualificados $(13,3 \%)$, dirigentes e administradores $(19,0 \%)^{7}$.

Apesar da tendência de declínio do tabagismo observado em estudos nos países desenvolvidos, entre as categorias de trabalhadores manuais essa redução foi pouco significativa comparada com a observada nos trabalhadores não manuais ${ }^{7,9}$. Smith considera que estes achados podem estar relacionados à renda e escolaridade, uma vez que nas categorias de trabalho não manual, o nível de escolaridade e de renda são maiores, fatores estes conhecidamente protetores para o tabagismo ${ }^{10}$. Além disso, o autor aponta que certas ocupações podem encorajar o início do fumo. Embora essas duas colocações tenham sido postuladas, não há evidências que confirmem o motivo das diferenças de tabagismo entre os diferentes grupos ocupacionais. Nossos resultados mostraram que, mesmo controlando para idade, sexo e renda, as diferenças entre grupos ocupacionais em relação ao tabagismo permanecem - reforçando a ideia de que há um efeito ligado à ocupação. $O$ fato é que identificar a população de fumantes segundo sua ocupação profissional pode contribuir na melhoria das intervenções relacionadas ao controle e à cessação do tabagismo, utilizando o ambiente de trabalho para tal.

Além do tabagismo no Brasil variar de acordo com a categoria de ocupação, fatores como turno de jornada de trabalho não diurno, emprego privado, carga horária semanal de trabalho maior e local do estabelecimento do trabalho aberto ou domiciliar estiveram associados ao maior consumo de cigarros. A literatura aponta 
que a convivência com colegas tabagistas e a jornada de trabalho estressante constituem fatores de risco para o fumo entre trabalhadores ${ }^{19}$. A maior instabilidade empregatícia gerando níveis de estresse aumentados poderia explicar a prevalência 31\% maior de tabagismo encontrada entre empregados do setor privado em relação ao setor público. Outro fator é que as ocupações com maiores prevalências são também aquelas em que o fumo não sofre medidas restritivas no local de trabalho, conforme legislação brasileira para ambientes coletivos privado ou público ${ }^{20-23}$.

Em resumo, confirmamos a tendência de redução da prevalência do fumo diário no Brasil, mostrada por inquéritos nacionais e pelo VIGI-
TEL. Ainda que esta redução tenha sido menor entre as mulheres, o tabagismo continua mais frequente em homens. As desigualdades regionais e as diferenças entre os estratos de renda permanecem um desafio às políticas de controle do tabagismo. O consumo diário do tabaco é maior entre trabalhadores que realizam tarefas manuais e nos empregados do setor privado, independente de sexo, renda e idade. A avaliação e o monitoramento do tabagismo em trabalhadores podem auxiliar na identificação dos grupos ocupacionais que necessitam prioridade nas intervenções, como medidas educativas no ambiente de trabalho, programas de redução de estresse e o acesso ao tratamento para cessação de fumar.

\section{Colaboradores}

AJD Barros, AM Cascaes, FC Wehrmeister, J Martínez-Mesa e AMB Menezes participaram igualmente de todas as etapas de elaboração do artigo. 


\section{Referências}

1. World Health Organization (WHO). WHO Report on the Global Tobacco Epidemic, 2008: The MPOWER package. Geneva: World Health Organization; 2008.

2. Lopez AD, Collishaw NE, Piha T. A descriptive model of the cigarette epidemic in developed countries. Tob Control 1994; 3(3):242-247.

3. World Health Organization (WHO). WHO Report on the Global Tobacco Epidemic, 2009: Implementing smoke-free environments. Geneva: World Health Organization; 2009.

4. Instituto Brasileiro de Geografia e Estatística (IBGE). Pesquisa Nacional por Amostra de Domicílios: Tabagismo 2008. Rio de Janeiro: Instituto Brasileiro de Geografia e Estatística; 2009.

5. Menezes AM, Minten GC, Hallal PC, Victora CG, Horta BL, Gigante DP, Barros FC. Smoking prevalence in the 1982 birth cohort: from adolescence to adult life, Pelotas, Southern Brazil. Rev Saude Publica 2008; 42(Supl. 2):78-85.

6. Nelson DE, Emont SL, Brackbill RM, Cameron LL, Peddicord J, Fiore MC. Cigarette smoking prevalence by occupation in the United States. A comparison between 1978 to 1980 and 1987 to 1990. J Occup Med 1994; 36(5):516-525.

7. Smith DR, Leggat PA. Tobacco smoking by occupation in Australia: results from the 2004 to $2005 \mathrm{Na}$ tional Health Survey. J Occup Environ Med 2007; 49(4):437-445.

8. Lee DJ, LeBlanc W, Fleming LE, Gomez-Marin O, Pitman T. Trends in US smoking rates in occupational groups: the National Health Interview Survey 1987-1994. J Occup Environ Med 2004; 46(6):538-548.

9. Lee DJ, Fleming LE, Arheart KL, LeBlanc WG, Caban AJ, Chung-Bridges K, Christ SL, McCollister KE, Pitman T. Smoking rate trends in U.S. occupational groups: the 1987 to 2004 National Health Interview Survey. J Occup Environ Med 2007; 49(1):75-81.

10. Smith DR. Tobacco smoking by occupation in Australia and the United States: a review of national surveys conducted between 1970 and 2005. Ind Health 2008; 46(1):77-89.

11. Instituto Brasileiro de Geografia e Estatística (IBGE). Síntese de indicadores 2008. Rio de Janeiro: Instituto Brasileiro de Geografia e Estatística; 2008. [acessado 2011 abr 11]. Disponível em: http://www.ibge.gov.br /home/estatistica/populacao/trabalhoerendimento/ pnad2008/

12. Barros AJ, Hirakata VN. Alternatives for logistic regression in cross-sectional studies: an empirical comparison of models that directly estimate the prevalence ratio. BMC Med Res Methodol 2003; 3:21.

13. Instituto Nacional do Câncer (INCA). Tabagismo no Brasil: um grave problema de saúde pública. Rio de Janeiro: Instituto Nacional do Câncer; 2007.
14. Pinto M, Uga MA. The cost of tobacco-related diseases for Brazil's Unified National Health System. Cad Saude Publica 2010; 26(6):1234-1245.

15. Travassos C, Viacava F, Laguardia J. Os Suplementos Saúde na Pesquisa Nacional por Amostra de Domicílios (PNAD) no Brasil. Rev Bras Epidemiol 2008; 11(Supl. 1):98-112.

16. Monteiro CA, Cavalcante TM, Moura EC, Claro RM, Szwarcwald CL. Population-based evidence of a strong decline in the prevalence of smokers in Brazil (1989-2003). Bull World Health Organ 2007; 85(7):527-534.

17. Instituto Brasileiro de Geografia e Estatística (IBGE). Pesquisa Nacional de Saúde do Escolar 2009. Rio de Janeiro: Instituto Brasileiro de Geografia e Estatística; 2009.

18. Malta DC, Moura EC, Silva SA, de Oliveira PP, Silva VL. Prevalence of smoking among adults residing in the Federal District of Brasilia and in the state capitals of Brazil, 2008. J Bras Pneumol 2010; 36(1):75-83.

19. Souza DPO, Silveira Filho DX. Uso recente de álcool, tabaco e outras drogas entre estudantes adolescentes trabalhadores e não trabalhadores. Revista Brasileira de Epidemiologia 2007; 10:276-87.

20. Brasil. Portaria n. ${ }^{\circ} 3.257$ de 22 de Setembro de 1988. Diário Oficial da União 1988; 22 set.

21. Brasil. Lei n. ${ }^{\circ} 9.294$ de 15 de Julho de 1996. Dispõe sobre as restrições ao uso e à propaganda de produtos fumígeros, bebidas alcoólicas, medicamentos, terapias e defensivos agrícolas, nos termos do $\$$ $4^{\circ}$ do art. 220 da Constituição Federal. Diário Oficial da União 1996; 15 jul.

22. Brasil. Decreto n. ${ }^{\circ} 2.018$ de $1^{\circ}$ de Outubro de 1996. Regulamenta a Lei no 9.294, de 15 de julho de 1996, que dispõe sobre as restrições ao uso e à propaganda de produtos fumígenos, bebidas alcoólicas, medicamentos, terapias e defensivos agrícolas, nos termos do $\$ 4^{\circ}$ do art. 220 da Constituição. Diário Oficial da União 1996; 1 out.

23. Brasil. Lei n. ${ }^{\circ} 10.167$ de 27 de Dezembro de 2000. Altera dispositivos da Lei no 9.294, de 15 de julho de 1996, que dispõe sobre as restrições ao uso e à propaganda de produtos fumígenos, bebidas alcoólicas, medicamentos, terapias e defensivos agrícolas. Diário Oficial da União 2000; 27 dez.

Artigo apresentado em 23/09/2010

Aprovado em 1/03/2011

Versão Final apresentada em 05/05/2011 